\title{
COLD1: a cold sensor in rice
}

\author{
SHI YiTing \& YANG ShuHua* \\ State Key Laboratory of Plant Physiology and Biochemistry, College of Biological Sciences, China Agricultural University, Beijing 100193, \\ China
}

Received February 24, 2015; accepted February 26, 2015; published online March 5, 2015

Citation: Shi YT, Yang SH. COLD1: a cold sensor in rice. Sci China Life Sci, 2015, 58: 409-410, doi: 10.1007/s11427-015-4831-6

Rice (Oryza sativa L.) is one of the most important food crops in the world. Originating in tropical and subtropical regions, rice cultivars are grouped into two major subspecies, indica (O. sativa ssp. indica) and japonica (O. sativa ssp. japonica). indica cultivars grown in low latitude areas are sensitive to low temperature, while japonica cultivars with more resistance to cold temperature are grown in temperate and frigid zones $[1,2]$. Low temperature is a major factor that affects rice production and distribution worldwide. Scientists have been making great efforts to identify genes that control chilling tolerance traits of rice. However, the molecular mechanism underlying the chilling adaptation of japonica is unknown.

Recently, the research group led by Kang Chong in Institute of Botany, Chinese Academy of Sciences successfully cloned a QTL named COLD1 (Chilling-Tolerance Divergence 1) [3]. One of the SNPs in this locus arisen during domestication confers chilling tolerance in japonica subspecies. This study also discovered that $C O L D I$ interacts with G-protein $\alpha$ subunit and functions in accelerating GTPase activity. Moreover, they provided elegant data showing that extracellular $\mathrm{Ca}^{2+}$ influx and net cytosolic $\mathrm{Ca}^{2+}$ concentration are mediated by COLDI in response to chilling stress. Their study, for the first time, uncovers a potential membrane-localized cold sensor in plants.

To identify chilling-tolerance-divergence (COLD), Ma et al. first generated recombinant inbred lines by crossing a chilling tolerant japonica cultivar Nipponbare (NIP) and a chilling-sensitive indica cultivar 93-11. Using molecular

*Corresponding author (email: yangshuhua@ cau.edu.cn) markers and QTL analysis, they defined COLD1 on chromosome 4. Fine mapping and DNA sequence comparisons revealed that a single nucleotide $\mathrm{A}$ at the 15 th nucleotide in the 4th exon of COLDI in NIP is substituted to T in 93-11, leading to a change in 187th amino acid from Lys to Met. The homozygous COLDI $1^{\text {NIP/NIP }}$ lines of near-isogenic lines (NILs) containing the $C O L D 1^{\mathrm{NIP}}$ locus in the 93-11 genetic background exhibit higher chilling tolerance than 93-11. Furthermore, the authors showed that $C O L D I^{\text {jap }}$-overexpression lines significantly enhance survival rates after chilling treatment compared to WT whereas cold1-1 mutant and COLDI antisense transgenic lines are chilling sensitive. These results suggest that $C O L D 1$ is a key gene modulating the chilling tolerance in japonica rice.

Importantly, Ma et al. discovered that a single-nucleotide polymorphism SNP2 in the 4th exon is associated with chilling tolerance. By sequencing the full-length COLD1 gene in 127 rice cultivars, they found that all japonica accessions and five $O$. rufipogon samples originating from China with stronger chilling tolerance have nucleotide $\mathrm{A}$ at the SNP2 sites. The chilling sensitive indica cultivars, however, have either T or C at the SNP2 site. From a geographical point of view, these japonica cultivars are mostly distributed in northeast Asia, the US, and higher elevation areas of the southeast Asia, whereas, indica cultivars are grown in southern and south-eastern Asia. Phylogenetic and nucleotide diversity analysis suggested that $\mathrm{SNP}^{\mathrm{A}}$ in COLD1 is likely to be derived from the Chinese O. rufipogon during japonica rice domestication.

Cold shock could activate the $\mathrm{Ca}^{2+}$ signaling pathway in plant cells, stimulating downstream cold signal transduction 
and transcription factor activation [4]. Little is known about the molecular mechanism of low temperature sensing and $\mathrm{Ca}^{2+}$ signaling. Ma et al. found that COLDl encodes a protein localized on plasma membrane and endoplasmic reticulum (ER). Like its orthologues GTG1/2 in Arabidopsis, COLD1 interacts with rice G-protein $\alpha$ subunit 1 (RGA1). However, COLD1 is different from GTG1/2 in intrinsic GTPase activity [5]. COLD1 alone does not have GTPase activity, but it can promote GTPase activity of RGA1. Therefore, COLD1 is a G-protein signaling regulator. Strikingly, they found that co-expressing COLDI $I^{\text {jap }}$ and $R G A 1$ rapidly activates inward current after cold treatment by using electrode voltage clamp approach. Furthermore, both $\mathrm{Ca}^{2+}$ influx and $\mathrm{Ca}^{2+}$ concentration in the cytoplasm showed a remarkable increase upon cold shock. Similar to TRPV which is an ion channel acting as a temperature sensor in mammalians, COLD1 is strongly suggested as a cold sensor in plants. In further study, it would be exciting to find out if COLD1 itself works as a potential calcium channel or a subunit of such ion channel.
In summary, Ma et al. reported the first plasma and ER-membrane protein involved in cold sensing in plants. Their findings contribute to our understanding of cold perception in plants. Meanwhile, using these NILs to confer chilling tolerance without negative effects on grain yield via molecular breeding techniques will definitely benefit human beings.

1 Kovach MJ, Sweeney MT, McCouch SR. New insights into the history of rice domestication. Trends Genet, 2007, 23: 578-587

2 Sang T, Ge S. Genetics and phylogenetics of rice domestication. Curr Opin Genet Dev, 2007, 17: 533-538

3 Ma Y, Dai X, Xu Y, Luo W, Zheng X, Zeng D, Pan Y, Lin X, Liu H, Zhang D, Xiao J, Guo X, Xu S, Niu Y, Jin J, Zhang H, Xu X, Li L, Wang W, Qian Q, Ge S, Chong K. COLD1 confers chilling tolerance in rice. Cell, 2015, doi: 10.1016/j.cell.2015.01.046

4 Knight H, Trewavas AJ, Knight MR. Cold calcium signaling in Arabidopsis involves two cellular pools and a change in calcium signature after acclimation. Plant Cell, 1996, 8: 489-503

5 Jaffe FW, Freschet GE, Valdes BM, Runions J, Terry MJ, and Williams LE. G protein-coupled receptor-type $G$ proteins are required for light-dependent seedling growth and fertility in Arabidopsis. Plant Cell, 2007, 24: 3649-3668

Open Access This article is distributed under the terms of the Creative Commons Attribution License which permits any use, distribution, and reproduction in any medium, provided the original author(s) and source are credited. 